\title{
Modesty and Security: Attributes Associated with Comfort and Willingness to Engage in Telelactation
}

\author{
Adetola F. Louis-Jacques ${ }^{1,2}$, Ellen J. Schafer ${ }^{3}{ }^{(}$, Taylor A. Livingston ${ }^{4}\left(\mathbb{D}\right.$, Rachel G. Logan ${ }^{5}$ and \\ Stephanie L. Marhefka ${ }^{5,6, *}$
}

1 Department of Obstetrics and Gynecology, Morsani College of Medicine, University of South Florida, Tampa, FL 33606, USA; alouisjacques@usf.edu

2 College of Nursing, University of South Florida, Tampa, FL 33612, USA

3 Department of Community and Environmental Health, College of Health Sciences, Boise State University, Boise, ID 83725, USA; ellenschafer@boisestate.edu

4 Department of Anthropology, University of Nebraska-Lincoln, Lincoln, NE 68588, USA; tlivingston3@unl.edu

5 College of Public Health, University of South Florida, Tampa, FL 33612, USA; logan23@usf.edu

6 The Chiles Center, University of South Florida, Tampa, FL 33613, USA

* Correspondence: smarhefk@usf.edu; Tel.: +1-813-974-5810; Fax: +1-813-974-5172

Citation: Louis-Jacques, A.F.; Schafer, E.J.; Livingston, T.A.; Logan, R.G.; Marhefka, S.L. Modesty and Security: Attributes Associated with Comfort and Willingness to Engage in Telelactation. Children 2021, 8, 271. https://doi.org/10.3390/children 8040271

Academic Editor: Elizabeth Asztalos

Received: 10 December 2020

Accepted: 26 March 2021

Published: 1 April 2021

Publisher's Note: MDPI stays neutral with regard to jurisdictional claims in published maps and institutional affiliations.

Copyright: (c) 2021 by the authors. Licensee MDPI, Basel, Switzerland. This article is an open access article distributed under the terms and conditions of the Creative Commons Attribution (CC BY) license (https:// creativecommons.org/licenses/by/ $4.0 /)$.

\begin{abstract}
The objectives were to identify conditions under which mothers may be willing to use telelactation and explore associations between participant characteristics, willingness, and beliefs regarding telelactation use. Mothers 2-8 weeks postpartum were recruited from two Florida maternal care sites and surveyed to assess demographics, breastfeeding initiation, and potential telelactation use. Analyses included descriptive statistics and logistic regression models. Of the 88 participants, most were white, married, earned less than USD 50,000 per year, had access to technology, and were willing to use telelactation if it was free $(80.7 \%)$ or over a secure server $(63.6 \%)$. Fifty-six percent were willing to use telelactation if it involved feeding the baby without a cover, but only $45.5 \%$ were willing if their nipples may be seen. Those with higher odds of willingness to use telelactation under these modesty conditions were experienced using videochat, white, married, and of higher income. Mothers with security concerns had six times the odds of being uncomfortable with telelactation compared to mothers without concerns. While telelactation can improve access to critical services, willingness to use telelactation may depend on conditions of use and sociodemographics. During the COVID-19 pandemic and beyond, these findings offer important insights for lactation professionals implementing virtual consultations.
\end{abstract}

Keywords: breastfeeding; telemedicine; lactation support; telelactation

\section{Introduction}

Breastfeeding is beneficial for infants and mothers [1-3]. As such, the American Academy of Pediatrics and the American College of Obstetricians and Gynecologists recommend exclusive breastfeeding of infants for six months and supplementation with complementary foods for at least one year [2,4]. Many women are unable to meet these recommendations and their breastfeeding goals due to challenges [5,6] including lack of social support, perceived insufficient milk supply, and latch difficulty $[7,8]$. Breastfeeding support services provided by a skilled lactation provider can address these challenges [9]. However, access to skilled lactation services is not geographically equitable in the United States [10]. For example, Florida, the third most populous state in the nation and the setting of this study, falls well below the recommended and national average of International Board Certified Lactation Consultants (IBCLC). While the recommended number of IBCLCs is 8.6 per 1000 births, Florida has only 2.55, compared to the national average of 3.86 per 1000 births [11]. 
Research has shown that telelactation, or the use of face-to-face videoconferencing software for lactation assistance via smartphone, tablet, or computer, is a promising option to provide professional lactation support [12-20], particularly for women in rural areas $[15,19,20]$. However, there are few studies on the acceptability of telelactation support $[13,14,17]$, and no studies on the acceptability of this technology among urban women from diverse demographic backgrounds. The primary objective of this study was to characterize technology use among urban mothers during the postpartum period and determine conditions under which women may be willing to adopt telelactation services and support. The secondary objective was to explore how participant characteristics impact willingness and beliefs regarding use of telelactation services. Data on conditions of use and sociodemographic differences in acceptability of telelactation services are particularly salient given the current SARS-CoV-2 pandemic as many women may be unwilling or unable to receive in-person lactation services.

\section{Materials and Methods}

\subsection{Procedures}

English- or Spanish-speaking women who were at least 18 years old and presenting for postpartum follow-up (between 2 and 8 weeks postpartum) were recruited to participate in the study at two maternal care sites in Tampa, FL. One recruitment site served mostly Medicaid patients while the other typically served those with private insurance, allowing a range of socioeconomic backgrounds. Women who did not have a live birth, whose children were admitted into the neonatal intensive care unit, or who were separated from their infants (e.g., foster care, adoption) were excluded from the study.

Clinic staff distributed survey packets to eligible participants. All contents of the packet were at a fifth-grade reading level and were available in English and in Spanish. Patients were instructed to read the contents of the survey packet, which included an introductory letter, informed consent, survey, future contact form, and gift card information. They were advised to contact research staff regarding any questions. Participants had the option to complete the survey via paper or online during the clinic visit or at a later time. The clinic staff member collected survey packets in sealed envelopes and placed the envelopes in a secure location. Participants who completed the survey via paper at a later time mailed the paper survey to the study office. Participants who chose to complete the survey online entered a link or scanned a $Q R$ code provided in the survey packet and completed all forms online.

The front desk staff distributed the survey packets to eligible participants (see File S1: "What Do Moms Need" Study Protocol). A total of 114 mothers provided consent and participated in a self-administered survey, receiving a USD 10 gift card for their time. Data were collected from November 2015 to June 2016. For the purpose of this study, only those who initiated breastfeeding and completed all survey questions $(N=88)$ were included in the analysis. All subjects gave their informed consent for inclusion before answering survey questions. The study was conducted in accordance with the Declaration of Helsinki, and the protocol was deemed exempt by the University of South Florida's Institutional Review Board (Pro000023145).

\subsection{Measures}

\subsubsection{Breastfeeding Initiation}

Mothers were defined as having initiated breastfeeding, and thus included in these analyses, if they responded "yes" to any combination of the following prompts: (1) In the hospital, I tried to feed my baby from the breast, (2) In the hospital, I tried to pump breast milk, or (3) In the hospital, my baby received some breast milk from me.

\subsubsection{Willingness to Use Telelactation Services}

Willingness to use telelactation was assessed by having mothers read a brief synopsis of a theoretical breastfeeding support application that would connect mothers to lacta- 
tion professionals (Figure 1: You and Technology) and then asked mothers to rate their willingness to use their mobile phones for this application through several Likert scale questions ( $1=$ definitely not to $5=$ definitely yes). Questions were developed with the goal of providing feedback for the purposes of developing a telelactation app. Questions were developed by the study team and reviewed for clarity and content by lactation professionals and obstetricians. The four questions used in this analysis were: "Would you be willing to use this professional breastfeeding support program if ..." (1) while breastfeeding the professional may see your nipples via videochat? (2) it involved the professional seeing you feeding your baby without a cover? (3) you knew it was over a secure server? (4) the whole program was free (Table 1)? These four items were dichotomized for analysis (probably yes or definitely yes vs. any other response).

YOU AND TECHNOLOGY

During this part of the survey, we want to learn of your opinions, thoughts and feelings about technology.

Imagine the time about 1 month before you had this baby. What if someone told you about a program that could allow you to have questions answered once you returned home. The program would give you access to professional breastfeeding support at home by using a videochat app on your smartphone.

This program would allow you to:

- $\quad$ Get info and help on-demand anywhere.

- Videochat with the help center anytime 24/7.

- $\quad$ Connect with a breastfeeding support professional via videochat.

- Have a hands-free session by using a phone holder (see picture on the right below).

The kind of help available could be:

- $\quad$ Education if you had a question about feeding your baby.

- Help such as how to get the baby to latch on.

- Other questions you may have about breastfeeding.

The trained professional would see you as you videochat about your breastfeeding questions. She would give you accurate info.

Figure 1. Brief synopsis of breastfeeding support application provided to participants.

Table 1. Willingness to use telelactation survey questions.

\begin{tabular}{|c|}
\hline \multirow{2}{*}{$\begin{array}{l}\text { Would You Be Willing to Use This Professional Breastfeeding Support Program If: } \\
\text { It cost } \$ 300 \text { a month and there would be little or no wait times for help }\end{array}$} \\
\hline \\
\hline It cost $\$ 210$ a month and there might be a $5-10$ min wait time for help \\
\hline The first session was free \\
\hline The professional spoke English but was from a different culture \\
\hline The professional spoke English but lived outside the United States \\
\hline Your doctor suggested it \\
\hline It provided info on local resources \\
\hline While breastfeeding the professional may see your nipples via videochat \\
\hline It involved the professional seeing you feeding your baby without a cover \\
\hline You knew it was over a secure server \\
\hline The whole program was free \\
\hline
\end{tabular}




\subsubsection{Beliefs Regarding Use of Telelactation Services}

Mothers were also asked about their beliefs regarding the telelactation application on their mobile devices with the following prompt: "The next set of questions ask about your feelings about using your mobile phone for professional breastfeeding support, just like you read about earlier. Please respond to each item below." Each of the following items were presented, and participants were asked to pick an option from a Likert-type scale $(1=$ strongly disagree to $5=$ strongly agree): (1) Videochat is good for some things, but not for getting help with breastfeeding. (2) If you use video-based breastfeeding support over a computer or Internet you can never be sure who will have access to the videochat session. (3) Videochat can provide access to breastfeeding support wherever women need it. (4) Women will not develop a personal relationship with a breastfeeding support professional if they only interact via videochat. (5) I do not feel comfortable with accessing video-based breastfeeding support online. These items were adapted from the Technology Readiness Index [21] and the Technology Readiness Index 2.0 [22] and were dichotomized for analysis ( $1=$ agree or strongly agree, $0=$ any other response). Other questions were presented in the survey but were not the focus of this analysis.

\subsection{Participant Characteristics}

We examined characteristics of the participants, including access to technology (computer, tablet, mobile phone with text messaging, and smart mobile phone); items were derived from the PEW Internet and American Life Project [23]. Past experience using videochat, age, marital status (married vs. any other response), employment status (working full- or part-time or self-employed vs. any other response), income (at least USD 50,000 per year vs. any other response), race (Black, white), and ethnicity (Hispanic vs. any other response) were assessed using investigator developed questions.

\section{Analyses}

Participant characteristics were explored using descriptive statistics. Separate bivariate logistic regression models were used to assess associations between participant characteristics and each of the nine outcome variables related to willingness to use telelactation and beliefs regarding telelactation services. To further explore mothers' comfort with accessing telelactation, the factors significantly associated in bivariate analysis with mothers agreeing to the statement "I do not feel comfortable with accessing video-based breastfeeding support online" were analyzed with multivariable logistic regression. A final model was derived by backward elimination. Lastly, we conducted a post hoc descriptive analysis to describe mothers who were simply not willing to use telelactation under any of the four conditions we asked about (i.e., if it's free, if nipples show, if nursing without a cover, and if over a secure server).

\section{Results}

Eighty-eight women who initiated breastfeeding and completed relevant items on the survey were included in the analyses. Most mothers were married, not currently working or self-employed, earned less than USD 50,000 per year, and identified as white. On average, participants were 29.3 years old $( \pm 5.05)$ with ages ranging from $18-44$ years. A majority of participants had access to each type of technology (computer, tablet, text messaging, smart phone); $94.3 \%$ indicated access to a smart phone and $53.4 \%$ indicated they had previously used the Internet for videochat. Participant characteristics are in Table 2. 
Table 2. Characteristics of the sample $(N=88)$.

\begin{tabular}{|c|c|}
\hline \multicolumn{2}{|l|}{ Characteristics } \\
\hline Age (years, mean \pm SD) & $29.3 \pm 5.05$ \\
\hline & $N(\%)$ \\
\hline Married & $48(54.5)$ \\
\hline Working (full or part time or self-employed) & $29(33.0)$ \\
\hline Income USD $50 \mathrm{~K}$ or more & $33(37.5)$ \\
\hline \multicolumn{2}{|l|}{ Race/Ethnicity * } \\
\hline Black $^{1}$ & $22(25.0)$ \\
\hline White ${ }^{1}$ & $45(51.1)$ \\
\hline Hispanic $^{1}$ & $22(25.0)$ \\
\hline \multicolumn{2}{|l|}{ Has access to ... } \\
\hline Desktop, laptop, netbook, or notebook computer & $72(81.8)$ \\
\hline Tablet computer (iPad, Samsung Galaxy, Windows Tablet) & $58(65.9)$ \\
\hline Mobile phone with text messaging & $82(93.2)$ \\
\hline Smart mobile phone (iPhone or Android) & $83(94.3)$ \\
\hline Use the Internet to videochat (Skype, Oovo, FaceTime) & $47(53.4)$ \\
\hline
\end{tabular}

Willing (probably yes or definitely yes vs. other responses) to use telelactation if ...

\begin{tabular}{|c|c|}
\hline The whole program was free & $71(80.7)$ \\
\hline You knew it was over a secure server & $56(63.6)$ \\
\hline It involved the professional seeing you feeding your baby without a cover & $49(55.7)$ \\
\hline While breastfeeding the professional may see your nipples via Videochat & $40(45.5)$ \\
\hline \multicolumn{2}{|l|}{$\begin{array}{l}\begin{array}{l}\text { Beliefs (agree or strongly agree vs. other responses) about using mobile phone } \\
\text { for telelactation }\end{array} \\
\end{array}$} \\
\hline Videochat can provide access to breastfeeding support wherever women need it & $57(64.8)$ \\
\hline $\begin{array}{l}\text { If you use video-based breastfeeding support over a computer or Internetyou can } \\
\text { never be sure who will have access }\end{array}$ & $43(48.9)$ \\
\hline I do not feel comfortable with accessing video-based breastfeeding support online & $23(26.1)$ \\
\hline Videochat is good for some things, but not for getting help with breastfeeding & $19(21.6)$ \\
\hline $\begin{array}{l}\text { Women will not develop a personal relationship with breastfeeding } \\
\text { support professional }\end{array}$ & $18(20.5)$ \\
\hline \multicolumn{2}{|l|}{ Use of (or willingness to use) other lactation support services } \\
\hline Website for baby-related information & $80(90.9)$ \\
\hline Text4Baby (a service that gives useful information to mothers via text) & $67(76.1)$ \\
\hline Breastfeeding consulting at clinic & $58(65.9)$ \\
\hline Breastfeeding help from a doula or traditional birth attendant & $58(65.9)$ \\
\hline Support group for new moms & $56(63.6)$ \\
\hline
\end{tabular}

${ }^{1}$ versus any other response. ${ }^{*}$ Participants could select more than one option.

\subsection{Willingness and Beliefs Regarding Telelactation Use}

As shown in Table 2, most participants were willing to use telelactation if the whole program was free $(80.7 \%)$, if they knew it was over a secure server $(63.6 \%)$, and if it involved feeding the baby without a cover (55.7\%). However, only $45.5 \%$ were willing to use telelactation if the professional may see their nipples via videochat. Despite $64.8 \%$ of participants agreeing with the belief that videochat can provide access to breastfeeding 
support wherever women need it, $48.9 \%$ of participants agreed with the belief that if you use video-based breastfeeding support via a computer or internet, you can never be sure who will have access. Upon exploring conditions under which women would use telelactation if the whole program was free (Figure 2), we found that 15 women would not use it under any conditions (see note for Figure 2).

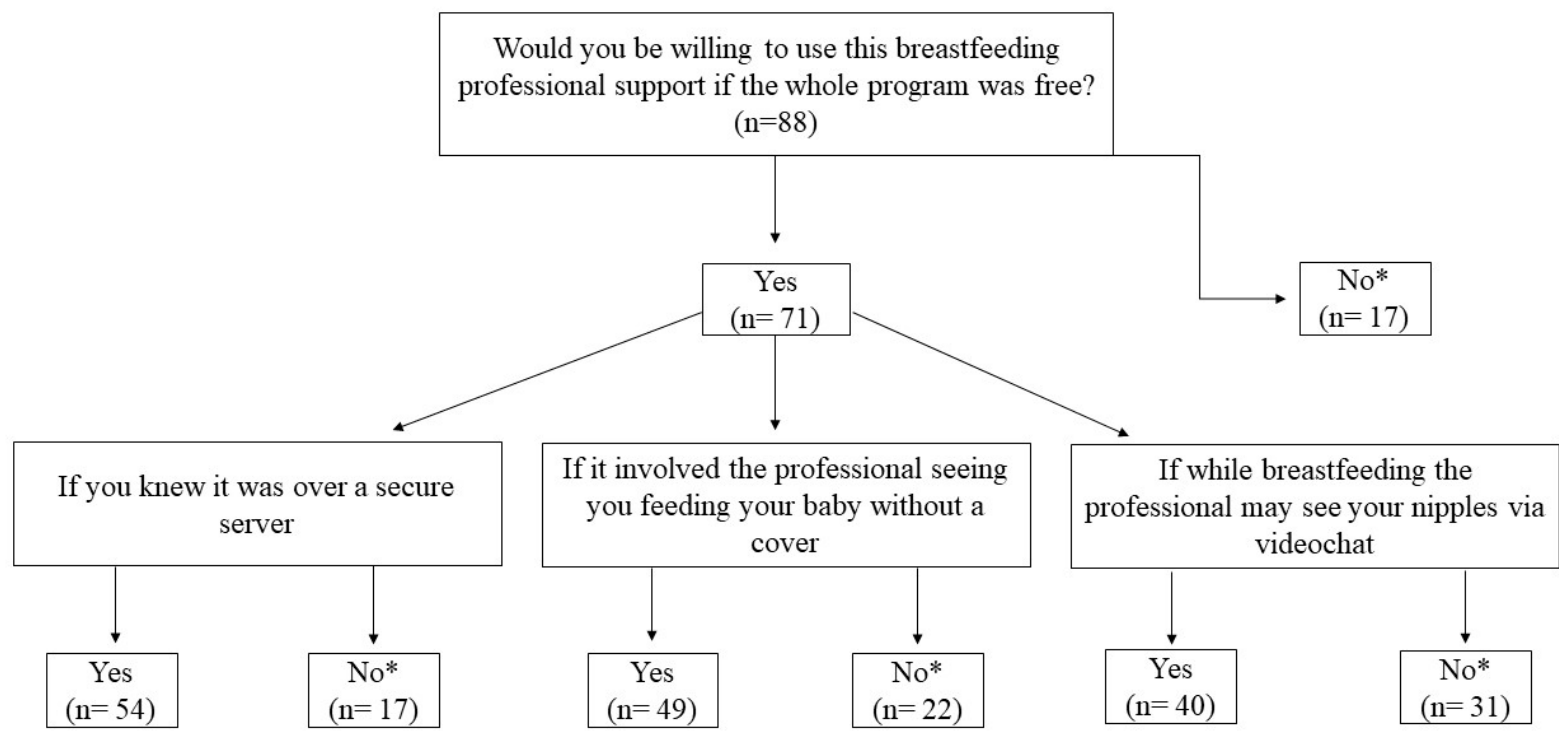

"Note 15 women said no to all willingness questions, indicating they were unwilling to use the proposed telelactation technology under any conditions. These mothers were also unwilling to receive lactation support in a clinic $(60 \%)$, via a doula/birth attendant $(60 \%)$; but most indicated they had used or were willing to use a support group for new moms (53\%), a text messaging app for information (73\%), or a website for baby-related information (73\%).

Figure 2. Conditions under which mothers would be willing to use telelactation services if it were free.

\subsection{Participant Characteristics and Willingness and Beliefs Variables}

We explored the willingness to use telelactation, beliefs about telelactation, and each demographic and technology access/use factor in bivariate analyses (see Table 3). Those with higher odds of being willing to use telelactation if the professional may see their nipples or if it meant feeding without a cover tended to be married, in the higher income category, white, and had experience using videochat. Those who indicated Black as their race had lower odds of willingness to use telelactation if it involved breastfeeding without a cover. Those with higher odds of being willing to use telelactation if they knew it was over a secure server tended to be married, in the higher income category, white, and had experience using videochat. Those who were married had greater odds, compared to those not married, to be willing to use telelactation if the program was free. Participants who were older, married, in the higher income category, and white had higher odds of agreeing with the belief that videochat can provide access to breastfeeding support wherever women need. White mothers were less likely to agree with the belief that "women will not develop a personal relationship with the breastfeeding support professional" if receiving breastfeeding support remotely. 
Table 3. Bivariate Associations between Participant Characteristics and Willingness or Beliefs Variables \#.

Willing (Probably Yes or Definitely Yes vs. Other Responses) to Use Telelactation If ...

\begin{tabular}{|c|c|c|c|c|c|c|}
\hline & $\begin{array}{c}\text { The Whole Program } \\
\text { Was Free }\end{array}$ & $\begin{array}{l}\text { You Knew It Was over a } \\
\text { Secure Server }\end{array}$ & $\begin{array}{l}\text { It Involved the } \\
\text { Professional Seeing } \\
\text { You Feeding Your Baby } \\
\text { without a Cover }\end{array}$ & $\begin{array}{l}\text { While Breastfeeding } \\
\text { the Professional May } \\
\text { See Your Nipples }\end{array}$ & $\begin{array}{l}\text { Videochat Can Provide } \\
\text { Access to Breastfeeding } \\
\text { Support Wherever } \\
\text { Women Need It }\end{array}$ & $\begin{array}{l}\text { Women Will Not Develop a } \\
\text { Personal Relationship with the } \\
\text { Breastfeeding } \\
\text { Support Professional }\end{array}$ \\
\hline & OR $(95 \%$ CI $)$ & OR $(95 \%$ CI $)$ & OR $(95 \%$ CI) & OR $(95 \%$ CI) & OR $(95 \%$ CI) & OR $(95 \%$ CI) \\
\hline Age & NS & NS & NS & NS & $1.17(1.05-1.30)^{* *}$ & NS \\
\hline Married $^{1}$ & $3.69(1.17-1.60) *$ & $7.50(2.79-20.15)^{* * *}$ & $6.23(2.46-15.79) * * *$ & $5.00(1.99-12.60)^{* *}$ & $4.20(1.65-10.68)^{* *}$ & NS \\
\hline Income $^{2}$ & NS & $4.03(1.44-11.31)^{* *}$ & $3.20(1.26-8.13)$ * & $3.32(1.35-8.16) * *$ & $5.02(1.69-14.92)^{* *}$ & NS \\
\hline Black $^{3}$ & NS & $0.36(0.14-0.98)$ * & $0.35(0.13-0.95)$ * & NS & NS & NS \\
\hline White $^{3}$ & NS & $4.60(1.79-11.83) * *$ & $3.77(1.55-9.15)^{* *}$ & $4.36(1.73-10.45)^{* *}$ & $2.69(1.09-6.65)$ * & $0.29(0.09-0.90)$ * \\
\hline Has used Videochat & NS & $3.44(1.28-8.55)^{* *}$ & $3.68(1.52-8.93)^{* *}$ & $2.39(1.01-5.67)$ * & NS & NS \\
\hline
\end{tabular}

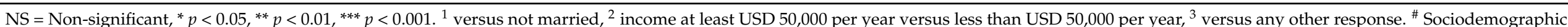

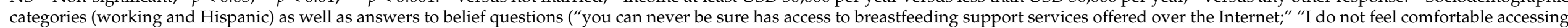
breastfeeding support online;" videochat is good for some things, but not for accessing breastfeeding support") were non-significant in all bivariate analyses, and thus omitted from the table.
Belief (Agree or Strongly Agree vs. Other Responses) about Using Mobile Phone for Telelactation

Women Will Not Develop

Personal Relationship

ort Professiona 


\subsection{Participant Characteristics and Discomfort}

To better define the $26.1 \%$ of participants who agreed they did not feel comfortable accessing video-based breastfeeding support online, we explored variables related to demographics, access to technology, and the other willingness and belief variables as predictors of not feeling comfortable with telelactation. The full model, in Table 4, includes those items that were associated with discomfort of using telelactation in bivariate analysis (willingness to use telelactation if: the professional may see your nipples, may see you feeding your baby without a cover, agreeing if you use video-based breastfeeding support you can never be sure who will have access, and agreeing videochat can provide access to breastfeeding support wherever women need it). There were no significant associations between discomfort and all demographic factors, all access to technology items, willingness to use telelactation if you knew it was over a secure server, willingness to use telelactation if the program was free, and agreeing that women will not develop a personal relationship with a breastfeeding support professional. The final model, in Table 4, shows mothers who agreed with concerns regarding who may have access to their telelactation session have more than six times the odds (OR $=6.32,95 \%$ CI: 1.82-21.98) of not being comfortable with telelactation compared to mothers who did not agree. Alternately, those who were willing to use telelactation if the breastfeeding professional might see their nipples $(\mathrm{OR}=0.20$, 95\% CI: 0.05-0.89) and agreed that videochat can provide access to breastfeeding support wherever women need it, had lower odds (OR $=0.29,95 \%$ CI: $0.08-0.99)$ of not feeling comfortable with accessing telelactation support.

Table 4. Multivariable model: factors related to mothers agreeing or strongly agreeing to the statement "I do not feel comfortable with accessing video-based breastfeeding support online".

\begin{tabular}{|c|c|c|}
\hline Attributes & $\begin{array}{l}\text { Full Model }{ }^{3} \\
\text { OR }(95 \% \text { CI })\end{array}$ & $\begin{array}{l}\text { Final Model } \\
\text { OR }(95 \% \text { CI })\end{array}$ \\
\hline Willing to use telelactation if while breastfeeding the professional may see your nipples ${ }^{1}$ & $0.19(0.03-1.04)$ & $0.20(0.05-0.89) *$ \\
\hline $\begin{array}{l}\text { Willing to use telelactation if it involved the professional seeing you feeding your baby without } \\
\text { a cover }{ }^{1}\end{array}$ & $1.18(0.28-4.87)$ & - \\
\hline $\begin{array}{l}\text { Agree if you use video-based breastfeeding support over a computer or Internet you can never be } \\
\text { sure who will have access }{ }^{2}\end{array}$ & $6.49(1.83-23.06)^{* *}$ & $6.32(1.82-21.98) * *$ \\
\hline Agree videochat can provide access to breastfeeding support wherever women need it ${ }^{2}$ & $0.28(0.08-0.98)^{*}$ & $0.29(0.08-0.99) *$ \\
\hline
\end{tabular}

\subsection{Post Hoc Analysis}

Fifteen mothers $(17 \%)$ were not willing to use telelactation under any condition (Figure 2). Most of these 15 mothers were not working $(80.0 \%)$, had an income lower than USD 50,000 per year $(80.0 \%)$, had never used videochat before $(60.0 \%)$, and owned a smart phone $(86.7 \%)$. The mothers unwilling to use telelactation were also unwilling to use (or had not previously used) lactation support: in a clinic $(60 \%)$ or via a doula/birth attendant $(60 \%)$; but most indicated they had used or were willing to use a support group $(53 \%)$, or a website $(73 \%)$ or text messaging $(73 \%)$ for baby-related information.

\section{Discussion}

In this study, we found that most mothers who initiated breastfeeding expressed willingness to use telelactation if it was free $(81 \%)$. Yet, prior to encountering the "free" question (which came last on the questionnaire), when they were asked about other conditions of telelactation use (if the professional could see the woman's nipples; if they would need to breastfeed over video without a cover; and if over a secure server), mothers' willingness was lower. Those more likely to be willing to use telelactation were married, in a higher income bracket, white, and had experience using videochat platforms, suggesting 
lower-income, non-white participants may have lower uptake of telelactation programs. These results differ from the only other quantitative study of the acceptability of telelactation, which found no significant differences in willingness to use the technology based on sociodemographic factors [14]. Our results suggest willingness to engage in telelactation may be contingent on a multitude of factors.

Other studies indicate that telelactation is a viable alternative to in-person lactation support for some populations [13-15,17,20]; however, our findings suggest it may not be acceptable to all. The complexity of acceptability of telelactation is supported by other findings from this study. Approximately $26 \%$ of women reported discomfort with the prospect of using telelactation. Greater discomfort was significantly associated with greater concerns about: (1) who may have access to the virtual session, (2) nipple exposure, and (3) disagreeing that telelactation can offer mothers access to support whenever they need it.

In contrast to our findings for willingness, we found that sociodemographic characteristics did not relate significantly to discomfort with telelactation use. It is possible that discomfort around telelactation might transcend sociodemographic groups, but willingness to adopt telelactation varies across sociodemographic groups. Black, lower income, and unmarried women have disparate breastfeeding outcomes and encounter many barriers and challenges in achieving their personal breastfeeding goals [24-28]. Our findings suggest that mothers who are unwilling to use telelactation might overlap with groups at risk for poor breastfeeding outcomes. Similarly, in a study of mothers within Mississippi's Women, Infant Children (WIC) program, only 7 percent downloaded a free telelactation app [29]. These findings suggest that as lactation services become more available on virtual platforms, it is critical that we find ways to support all breastfeeding mothers so that we do not further widen breastfeeding inequities. Breastfeeding inequities are a public health priority because they may contribute to other inequities in maternal and child health outcomes [28,30].

Fifteen mothers were unwilling to use telelactation under any condition. Most of these mothers were unemployed and of lower income, increasing their risk for poor breastfeeding outcomes [26]. Most mothers in this category had a smart phone but had never used videochat. This finding is consistent with another study showing people who had not used certain technologies were less likely to be willing to use those technologies to receive health-related information, even if the technology/service was free and accessible [31].

The mothers unwilling to use telelactation under the conditions investigated in this study were also unwilling to receive lactation support in person; but most were willing to use a website or text-messaging for baby-related information. It is important to understand how to best support this group of mothers who could be at high risk for early breastfeeding cessation [26]. Providing access to text-messaging or online patient-friendly breastfeeding resources such as videos on latch and breastfeeding positions or materials on breastfeeding challenges and management may be helpful to these mothers [32-34]. Further research exploring concerns and barriers regarding in person or virtual lactation support could help in development of resources.

The SARS CoV-2 (COVID-19 disease) pandemic and public health guidance on mitigation efforts have created new breastfeeding challenges. For example, the Centers for Disease Control and Prevention (CDC) recommends [35] determining on a "case-by-case basis" if a mother with suspected or confirmed COVID-19 should be separated from her newborn. In addition to the potentially unnecessary [36] separation of the breastfeeding dyad, other stressors may increase the need for lactation support. These stressors include COVID-19 anxiety [37], potential lack of support persons (including doulas) present during and after labor [38,39], possible redirected resources [40] and expedited discharge from the hospital [41] leading to missed breastfeeding education opportunities, as well as social isolation caused by physical distancing [35]. As breastfeeding is an important aspect of public health [42] with lifelong benefits for mother and child [43], it is critical that mothers receive the support they need in this unprecedented time. While many skilled lactation 
specialists have moved to the virtual realm to provide these necessary support services, our results indicate telelactation is not a "one size fits all" solution.

Indeed, findings suggest that lactation professionals may wish to adjust their practice to accommodate the comfort of each mother. When scheduling a telelactation visit, lactation providers may wish to provide anticipatory guidance-over the telephone, on their website or social media, or through information sent via text or email. This guidance should detail the role of the telelactation professional, as well as the potential activities involved in telelactation. Using the principles of shared decision-making [44], anticipatory guidance should be reviewed with the mother before the session, with plans regarding activities that will be conducted during the session. Ideally, providers will inform mothers that they will determine what happens in the telelactation session, based on their level of comfort and willingness to engage in certain activities (specifically showing their nipples, which is not always required in lactation visits). Additionally, providers should take care to: assure mothers that telelactation sessions will not be recorded or saved on any server, conduct sessions in private rooms (i.e., not in open areas of their home or office where other people may see the telelactation session [45]), and use HIPAA-compliant platforms when feasible [46] (see Table 5 for all recommendations).

Table 5. Key points of consideration regarding telelactation.

\begin{tabular}{ll}
\hline - & Provide anticipatory guidance upfront so mothers know what to expect during consultation \\
\hline - & Assure mothers the sessions are not recorded \\
\hline - & Adjust practice in consideration of mother's comfort level \\
& $\begin{array}{l}\text { Think creatively about working around discomfort and technological capabilities (access to } \\
\text { creative use of mirrors to show additional angles) }\end{array}$ \\
\hline - & Consider making videos using dolls or sending mothers other video resources with solutions \\
& to common breastfeeding problems (latch issues, milk production) to assist with education \\
\hline - & $\begin{array}{l}\text { Use HIPAA compliant and secure video conferencing platform, if available; providers } \\
\text { should use private location with minimal background for appointment }\end{array}$ \\
\hline - & $\begin{array}{l}\text { Conduct sessions alone in private rooms and consider locking the door, to ensure no other } \\
\text { person in the provider's environment will compromise the mother's privacy }\end{array}$ \\
\hline
\end{tabular}

Understanding and expanding the use of technology for breastfeeding support even after the COVID-19 pandemic may be important in helping mothers sustain breastfeeding, as research has shown women who used telelactation had increased exclusive breastfeeding and longer durations of breastfeeding than women who only had access to in-person lactation services $[20,29]$. Women in our study who perceived telelactation as offering support to women "wherever they needed it" were less likely to feel uncomfortable using telelactation, suggesting that marketing this attribute may be effective in increasing uptake. Lactation professionals have a positive effect on breastfeeding exclusivity and duration $[47,48]$, and telelactation, which allows for live counseling to mothers outside of normal clinical hours, offers a less time consuming and more accessible [13] way for women to attain breastfeeding support than in person consultations.

\section{Strengths and Limitations}

This study provides relevant, timely information on how to support breastfeeding mothers using telelactation and points to considerations that may impact initial uptake or sustained engagement with telelactation support. We sampled a diverse group of women receiving care in urban maternal care sites. However, our study has limitations, including a limited sample size and the consequential limited power to detect statistically significant differences. Indeed, the initial goal of the study was to conduct formative research to inform the development of a mobile telelactation app, though we still found significant associations. As such, while a larger sample would allow for greater exploration of willingness to use 
and comfort with telelactation, the results of this study are still relevant for those who are interested in working with women via telelactation. Additionally, measurement of the construct "willingness" may not fully encompass the barriers, including structural barriers, to women accessing and feeling comfortable using telelactation. Future research should qualitatively explore women's willingness to use telelactation services in order to ascertain barriers and ways to assuage discomfort with its usage. Further, additional studies are needed to investigate the willingness to use and comfort with telelactation services for women not included in our sample, including those whose infants spend time in the neonatal intensive care unit, separated from the infant, and/or were too ill or unable to initiate breastfeeding. Finally, the data were collected in 2015 and 2016, prior to the COVID-19 pandemic. Mothers may be more willing to use lactation support during the pandemic given transmission concerns regarding non-physically distanced care. However, our results provide relevant, timely information on how to support breastfeeding mothers using telelactation and points to sustained concerns (pandemic or not) that may impact initial uptake or engagement with telelactation support.

\section{Conclusions}

Telelactation is perceived by some as a viable alternative to in-person lactation support; however, it may not be acceptable to all. Future research should identify ways to engage mothers who have concerns with telelactation use and continue efforts to increase access to and comfort with breastfeeding support through technology. Such engagements should particularly focus on innovative ways to support mothers who are uncomfortable using telelactation.

Author Contributions: Conceptualization, A.F.L.-J., R.G.L., S.L.M.; Methodology, A.F.L.-J., R.G.L., S.L.M.; Formal Analysis, E.J.S., T.A.L.; Investigation, S.L.M.; Data Curation, R.G.L., E.J.S.; WritingOriginal Draft Preparation, A.F.L.-J., E.J.S., T.A.L., R.G.L., S.L.M.; Writing-Review and Editing, A.F.L.-J., E.J.S., T.A.L., R.G.L., S.L.M.; Visualization, A.F.L.-J., E.J.S., T.A.L., R.G.L., S.L.M.; Supervision, S.L.M.; Project Administration, S.L.M., A.F.L.-J., R.G.L.; Funding Acquisition, S.L.M. All authors have read and agreed to the published version of the manuscript.

Funding: We would like to thank Andrew Romaner for his financial support of the project. The lead author of this work was supported by the Foundation for SMFM/AAOGF scholar award.

Institutional Review Board Statement: The study was conducted in accordance with the Declaration of Helsinki. Ethical review and approval were waived for this study by the University of South Florida's Institutional Review Board (Pro000023145). The study activities were deemed to be a needs assessment of resources for nursing mothers and the activities did not require USF IRB oversight.

Informed Consent Statement: Informed consent was obtained from all subjects involved in the study.

Data Availability Statement: The data presented in this study are available on request from the corresponding author.

Acknowledgments: We would like to thank Andrew Romaner for his assistance with development of the study concept. Two abstracts were presented based on this work: (1) "Uncovering" the truth about technology-based breastfeeding support: Barriers to video-chat lactation-consultation (VCLC) uptake was a poster presentation at the Academy of Breastfeeding Medicine 21st Annual International Conference, October 2016. (2) iBreastfeed: Moms' willingness to receive technology-augmented lactation consultation was an oral presentation at the American Public Health Association 144th Annual Meeting and Expo, November 2016.

Conflicts of Interest: The authors declare no conflict of interest.

\section{References}

1. Louis-Jacques, A.; Stuebe, A. Long-term maternal benefits of breastfeeding: Longer durations of breastfeeding are associated with improved health outcomes for mothers and should be supported by ob/gyns. J. Contemp. OB GYN 2018, 63, 26-30.

2. BEidelman, A.I.; Schanler, R.J. Section on Breastfeeding. Breastfeeding and the use of human milk. Pediatrics 2012, 129 , e827-e841. [CrossRef] 
3. Feltner, C.; Weber, R.P.; Stuebe, A.; Grodensky, C.A.; Orr, C.; Viswanathan, M. Breastfeeding Programs and Policies, Breastfeeding Uptake, and Maternal Health Outcomes in Developed Countries; Agency for Healthcare Research and Quality: Rockville, MD, USA, 2018.

4. Breastfeeding Expert Work Group; American College of Obstetricians \& Gynecologists Committee on Obstetric Practice. Committee Opinion No. 658: Optimizing support for breastfeeding as part of obstetric practice. J. Obstet. Gynecol. 2016, 127, e86. [CrossRef] [PubMed]

5. Schafer, E.J.; Buch, E.D.; Campo, S.; Ashida, S. From initiation to cessation: Turning points and coping resources in the breastfeeding experience of first-time mothers. Women Health 2019, 59, 449-463. [CrossRef]

6. Hinsliff-Smith, K.; Spencer, R.; Walsh, D. Realities, difficulties, and outcomes for mothers choosing to breastfeed: Primigravid mothers experiences in the early postpartum period (6-8 weeks). Midwifery 2014, 30, e14-e19. [CrossRef]

7. Hornsby, P.P.; Gurka, K.K.; Conaway, M.R.; Kellams, A.L. Reasons for early cessation of breastfeeding among women with low income. Breastfeed. Med. 2019, 14, 375-381. [CrossRef]

8. Morrison, A.H.; Gentry, R.; Anderson, J.J. Mothers' Reasons for Early Breastfeeding Cessation. Am. J. Matern. Child Nurs. 2019, 44, 325-330. [CrossRef]

9. Teich, A.S.; Barnett, J.; Bonuck, K. Women's perceptions of breastfeeding barriers in early postpartum period: A qualitative analysis nested in two randomized controlled trials. Breastfeed. Med. 2014, 9, 9-15. [CrossRef] [PubMed]

10. Grubesic, T.H.; Durbin, K.M. Breastfeeding support: A geographic perspective on access and equity. J. Hum. Lact. 2017, 33, 770-780. [CrossRef]

11. Eden, A.R.; Anstey, E.H.; Orriola, D.J. Growing the IBCLC workforce: A Florida needs assessment. Hum. Lact. 2018, 34, 148-155. [CrossRef] [PubMed]

12. Friesen, C.A.; Hormuth, L.J.; Petersen, D.; Babbitt, T. Using videoconferencing technology to provide breastfeeding support to low-income women: Connecting hospital-based lactation consultants with clients receiving care at a community health center. J. Hum. Lact. 2015, 31, 595-599. [CrossRef] [PubMed]

13. Habibi, M.F.; Nicklas, J.; Spence, M.; Hedberg, S.; Magnuson, E.; Kavanagh, K.F. Remote lactation consultation: A qualitative study of maternal response to experience and recommendations for survey development. J. Hum. Lact. 2012, $28,211-217$. [CrossRef] [PubMed]

14. Habibi, M.F.; Springer, C.M.; Spence, M.L.; Hansen-Petrik, M.B.; Kavanagh, K.F. Use of Videoconferencing for Lactation Consultation: An Online Cross-Sectional Survey of Mothers' Acceptance in the United States. J. Hum. Lact. 2018, 34, 313-321. [CrossRef]

15. Kapinos, K.; Kotzias, V.; Bogen, D.; Ray, K.; Demirci, J.; Rigas, M.A.; Uscher-Pines, L. The Use of and Experiences With Telelactation Among Rural Breastfeeding Mothers: Secondary Analysis of a Randomized Controlled Trial. J. Med. Internet Res. 2019, 21 , e13967. [CrossRef]

16. Marcucci, B. Use of Telehealth to Increase Breastfeeding Exclusivity and Duration. Clin. Lact. 2018, 9, 66-71. [CrossRef]

17. Rojjanasrirat, W.; Nelson, E.-L.; Wambach, K.A. A pilot study of home-based videoconferencing for breastfeeding support. J. Hum. Lact. 2012, 28, 464-467. [CrossRef]

18. Uscher-Pines, L.; Mehrotra, A.; Bogen, D.L. The emergence and promise of telelactation. Am. J. Obstet. Gynecol. 2017, 217, 176-178.e171. [CrossRef]

19. Demirci, J.; Kotzias, V.; Bogen, D.L.; Ray, K.N.; Uscher-Pines, L. Telelactation via mobile app: Perspectives of rural mothers, their care providers, and lactation consultants. Telemed. Health 2019, 25, 853-858. [CrossRef]

20. Uscher-Pines, L.; Ghosh-Dastidar, B.; Bogen, D.L.; Ray, K.N.; Demirci, J.R.; Mehrotra, A.; Kapinos, K.A. Feasibility and Effectiveness of Telelactation Among Rural Breastfeeding Women. Acad. Pediatrics 2019. [CrossRef]

21. Parasuraman, A. Technology Readiness Index (TRI) a multiple-item scale to measure readiness to embrace new technologies. $J$. Serv. Res. 2000, 2, 307-320. [CrossRef]

22. Parasuraman, A.; Colby, C.L. An Updated and Streamlined Technology Readiness Index:TRI 2.0. J. Serv. Res. 2015, 18, 59-74. [CrossRef]

23. Project, P. Pew Internet and American Life Project. Available online: https://www.loc.gov/item/lcwaN0003976/ (accessed on 21 May 2020).

24. Sriraman, N.K.; Kellams, A. Breastfeeding: What are the Barriers? Why Women Struggle to Achieve Their Goals. J. Women Health 2016, 25, 714-722. [CrossRef] [PubMed]

25. Centers for Disease Control and Prevention. Breastfeeding among U.S. Children Born 2000-2016, CDC National Immunization Survey. Available online: https:/ /www.cdc.gov/breastfeeding/data/nis_data/results.html (accessed on 1 December 2020).

26. Centers for Disease Control and Prevention. Rates of Any and Exclusive Breastfeeding by Socio-demographics among Children Born in 2016. Available online: https:/ / www.cdc.gov/breastfeeding/data/nis_data/rates-any-exclusive-bf-socio-dem-2016.htm (accessed on 14 December 2019).

27. Deubel, T.F.; Miller, E.M.; Hernandez, I.; Boyer, M.; Louis-Jacques, A. Perceptions and Practices of Infant Feeding among African American Women. Ecol. Food Nutr. 2019, 58, 301-316. [CrossRef] [PubMed]

28. Louis-Jacques, A.; Deubel, T.F.; Taylor, M.; Stuebe, A.M. Racial and ethnic disparities in U.S. breastfeeding and implications for maternal and child health outcomes. Semin. Perinatol. 2017, 41, 299-307. [CrossRef] 
29. Hunt, A.T. Telelactation and Breastfeeding Outcomes among Low-Income Mothers in Mississippi: A Retrospective Cohort Study; ProQuest 2018; Dissertation University of Nevada: Las Vegas, NV, USA, 2018.

30. Bartick, M.C.; Jegier, B.J.; Green, B.D.; Schwarz, E.B.; Reinhold, A.G.; Stuebe, A.M. Disparities in Breastfeeding: Impact on Maternal and Child Health Outcomes and Costs. J. Pediatrics 2017, 181, 49-55.e46. [CrossRef]

31. Marhefka, S.L.; Lockhart, E.; Turner, D.; Wang, W.; Dolcini, M.M.; Baldwin, J.A.; Roig-Romero, R.M.; Lescano, C.M.; Glueckauf, R.L. Social Determinants of Potential eHealth Engagement Among People Living with HIV Receiving Ryan White Case Management: Health Equity Implications from Project TECH. AIDS Behav. 2020, 24, 1463-1475. [CrossRef]

32. Ahmed, A.; Ouzzani, M. Development and assessment of an interactive web-based breastfeeding monitoring system (LACTOR). Matern. Child Health J. 2013, 17, 809-815. [CrossRef]

33. American College of Obstetricians and Gynecologists. Breastfeeding Toolkit. 2016. Available online: https://www.acog.org/-/ media/project/acog/acogorg/files/pdfs/publications/breastfeedingtoolkit2020.pdf (accessed on 3 March 2021).

34. Office on Women's Health in the U.S. Department of Health and Human Services. Breastfeeding. Available online: https: / / www.womenshealth.gov / breastfeeding (accessed on 29 May 2020).

35. National Center for Immunization and Respiratory Diseases (NCIRD), Centers for Disease Control and Prevention. Coronavirus Disease 2019: Considerations for Inpatient Obstetric Healthcare Settings. Available online: https: / /www.cdc.gov/coronavirus / 2019-ncov/hcp/inpatient-obstetric-healthcare-guidance.html (accessed on 16 April 2020).

36. Stuebe, A. Should Infants Be Separated from Mothers with COVID-19? First, Do No Harm. Breastfeed. Med. 2020, 15. ahead of print. [CrossRef]

37. Davenport, M.H.; Meyer, S.; Meah, V.L.; Strynadka, M.C.; Khurana, R. Moms Are Not OK: COVID-19 and Maternal Mental Health. Front. Glob. Women Health 2020, 1, 1. [CrossRef]

38. Hermann, A.; Deligiannidis, K.M.; Bergink, V.; Monk, C.; Fitelson, E.M.; Robakis, T.K.; Birndorf, C. Response to SARS-Covid19-related visitor restrictions on labor and delivery wards in New York City. Arch. Women Ment. Health 2020, 23, 793-794. [CrossRef]

39. Fetters, A. COVID-19 Is Upending Parents' Birth Plans. In The Atlantic; Emerson Collective: Palo Alto, CA, USA, 2020.

40. Riley, T.; Sully, E.; Ahmed, Z.; Biddlecom, A. Estimates of the potential impact of the COVID-19 pandemic on sexual and reproductive health in low-and middle-income countries. Int. Perspect. Sex. Reprod. Health 2020, 46, 46. [CrossRef] [PubMed]

41. Capanna, F.; Haydar, A.; McCarey, C.; Bernini Carri, E.; Bartha Rasero, J.; Tsibizova, V.; Helmer, H.; Makatsarya, A.; Di Renzo, G. Preparing an obstetric unit in the heart of the epidemic strike of COVID-19: Quick reorganization tips. J. Matern. Fetal Neonatal Med. 2020, 1-7. [CrossRef] [PubMed]

42. Brown, A. Breastfeeding as a public health responsibility: A review of the evidence. J. Hum. Nutr. Diet. 2017, 30, 759-770. [CrossRef] [PubMed]

43. Victora, C.G.; Bahl, R.; Barros, A.J.; França, G.V.; Horton, S.; Krasevec, J.; Murch, S.; Sankar, M.J.; Walker, N.; Rollins, N.C. Breastfeeding in the 21st century: Epidemiology, mechanisms, and lifelong effect. Lancet 2016, 387, 475-490. [CrossRef]

44. Elwyn, G.; Frosch, D.; Thomson, R.; Joseph-Williams, N.; Lloyd, A.; Kinnersley, P.; Cording, E.; Tomson, D.; Dodd, C.; Rollnick, S. Shared decision making: A model for clinical practice. J. Gen. Intern. Med. 2012, 27, 1361-1367. [CrossRef]

45. Marhefka, S.; Lockhart, E.; Turner, D. Achieve Research Continuity During Social Distancing by Rapidly Implementing Individual and Group Videoconferencing with Participants: Key Considerations, Best Practices, and Protocols. AIDS Behav. 2020. [CrossRef]

46. Office for Civil Rights, D. Notification of Enforcement Discretion for Telehealth Remote Communications during the COVID19 Nationwide Public Health Emergency. Available online: https:/ /www.hhs.gov/hipaa/for-professionals/special-topics / emergency-preparedness/notification-enforcement-discretion-telehealth/index.html (accessed on 16 April 2020).

47. Chetwynd, E.M.; Wasser, H.M.; Poole, C. Breastfeeding Support Interventions by International Board Certified Lactation Consultants: A Systemic Review and Meta-Analysis. J. Hum. Lact. 2019, 35, 424-440. [CrossRef]

48. Haase, B.; Brennan, E.; Wagner, C.L. Effectiveness of the IBCLC: Have we Made an Impact on the Care of Breastfeeding Families Over the Past Decade? J. Hum. Lact. 2019, 35, 441-452. [CrossRef] [PubMed] 